\title{
Influence of fungicides on toxigenic properties of phytopathogenic fungi
}

\author{
Ekaterina $V$. Baybakova ${ }^{1}$, Elena E. Nefed'eva ${ }^{1,2, *}$, Maria N. Belitskaya ${ }^{3}$, Irina R. Gribust ${ }^{3}$, \\ Margarita D. Kosogorina ${ }^{1}$, and Galina A. Sevriukova ${ }^{1}$ \\ ${ }^{1}$ Volgograd State Technical University, Department of Industrial Ecology and Safety, 400005 \\ Volgograd, Russia \\ ${ }^{2}$ Volgograd State University, Department of Biology, 400062 Volgograd, Russia \\ ${ }^{3}$ Federal Scientific Centre of Agroecology, Complex Meliorations and Protective Afforestation of the \\ Russian Academy of Sciences, 400062 Volgograd, Russia
}

\begin{abstract}
Fusarium infection promotes losses of yield and death of plants. Fusarium infections are dangerous because grains contain fusariotoxins that cause intoxication in humans and animals, as well as necrosis of plant tissues and changes in plant growth. Fungicides inhibit the growth of mycelium, but strobilurins are known to promote the production of mycotoxins by fungi, and triazoles, on the contrary, reduce the production of deoxynivalenol. The toxigenic effect of fungicides was assessed by biotest method. Wheat grains were germinated with the addition of disinfected culture fluid. The culture fluid after the cultivation of Fusarium oxysporum with addition of azoxystrobin and pyraclostrobin in small doses was toxic for grains. The death of a significant part of the grains was noted in it. The fungistatic effect of small doses was not expressed. Therefore, the adaptation of the fungus was realized as a change in metabolism. High doses of azoxystrobin and pyraclostrobin in culture fluid increased seed germination. Those doses had a fungistatic effect. The addition of carbendazim increased the fungistatic effect and decreased the toxicity of the strain. Development of combined fungicides with sufficient doses of active substances reduces the risk of inhibition of the growth of the protected crops.
\end{abstract}

\section{Introduction}

Fungi of the genus Fusarium are characterized by high metabolic activity and plasticity to environmental conditions. Along with the saprophytic nutrition, they are parasitic on various plants and even animals [1]. Fungi of the genus Fusarium are the causes of disease in vegetables and crops. In cereals, they appear on spikelets and grains, manifesting themselves in an explicit and latent form. This causes significant damage to yield and the quality of product. Fusarium infection leads to significant losses in yield and plant death. In addition, fusarium infections are dangerous because grains that are normal in appearance contain fusariotoxins. They cause digestive and neurotoxic disorders of varying severity in humans and animals [2], and also cause necrosis of plant tissues and affect plant growth [3].

\footnotetext{
*Corresponding author: nefedieva@rambler.ru
} 
The danger of fusariotoxins to the health of human and farm animals is recognized by the international community. Fatal acute intoxication of humans and animals with fungal metabolites, leading to death are known in different countries. Chronic mycotoxin intoxication, which disrupts the functioning of internal organs, also poses a serious danger. Acute or latent intoxication by grain contaminated with mycotoxins is a constant serious problem in livestock and poultry farming as well [4].

Mycotoxins vary in degree of danger, which depends on the method and period of their entry into the body, on the type of animal, its age, gender and individual differences. Many countries and international organizations are revising regulatory documents, setting limit values for their contents to ensure maximum safety of food and fodder. This is a very difficult process, which is influenced by various factors, including the political, economic, commercial interests of each country, as well as the insufficient methodological ability to conduct large-scale analyses [5].

For example, in the Russian Federation, maximum permissible concentrations of three fusariotoxins are established. They variation depends on the type and purpose of grain production: deoxynivalenol (DON) $-0.7 \ldots 1 \mathrm{mg} / 1 \mathrm{~kg}$, T2 toxin $-0.1 \mathrm{mg} / 1 \mathrm{~kg}, \mathrm{ZEA}-0.2$ $-1 \mathrm{mg} / 1 \mathrm{~kg}$ of grain raw materials in accordance with the standards of food and feed product safety control. Mycotoxins are not allowed in baby and diet foods [6].

DON is a mycotoxin produced by fungi of the genus Fusarium. Maximum tolerable DON concentrations for grain and selected wheat products were laid down by national and European legislative institutions to protect consumers from health risks associated with the intake of this mycotoxin [7].

Phytopathogenic fungi can produce phytohormone abscisic acid (ABA) which plays significant role in many physiological processes and response of plants to abiotic and biotic stresses. Unfortunately, the role of this trait in interactions with host plants is poorly understood. Production of ABA depends on the strain of phytopathogenic fungi as well as on media [8].

Fungicides inhibit the growth of mycelium, but strobilurins are known to promote the production of mycotoxins by fungi [9].

Among the factors that can be controlled by growers, previous crop, tillage, wheat cultivar and fungicide regimes were identified as the most important variables for DON contamination of wheat. Careful choice of cultivar is currently the most effective agronomic method to decrease DON contamination levels in wheat followed by ploughing, avoiding maize as pre-crop and applying triazole fungicides at wheat anthesis [7].

The work was aimed to clarify the inhibitory effect of the active substances (a.s.) carbendazim, azoxystrobin and pyraclostrobin, which belong to different chemical classes on plant growth and toxigenic effect on phytopathogenic fungi. Researches of the effect of a mixture of different doses of a.s. in possible ratios were carried out.

\section{Materials and methods}

The strain MFG58518 Fusarium oxysporum, kindly given by the laboratory of Mycology and Phytopathology of All-Russian institute of plant protection (FSBSI VIZR), was used. The strain was cultured in Petri dishes on the dense nutrient medium of Czapek. The dishes were illuminated with a $30 \mathrm{~W}$ erythema lamp to induce sporulation.

Phytopathogenic fungi was cultivated in static culture as follows. $100 \mu$ of suspension of conidia of fungus that contains $10^{3} \mathrm{CFU}$ was inoculated into sterile flasks with $50 \mathrm{ml}$ of sterile liquid medium. In the same flasks, solutions of fungicides were introduced so that their concentration in the medium was $1 ; 3$ and 5 ppm. We made up mix options of carbendazim and azoxystrobin in ratios $0: 0 ; 0: 1 ; 0: 3 ; 0: 5 ; 1: 0 ; 1: 1 ; 1: 3 ; 1: 5 ; 3: 0 ; 3: 1 ; 3: 3 ; 3: 5 ; 5: 0 ; 5: 1 ; 5: 3$; $5: 5$. We made up mix options of carbendazim and pyraclostrobin in ratios $0: 0 ; 0: 1 ; 0: 3 ; 0: 5$; 
$1: 0 ; 1: 1 ; 1: 3 ; 1: 5 ; 3: 0 ; 3: 1 ; 3: 3 ; 3: 5 ; 5: 0 ; 5: 1 ; 5: 3 ; 5: 5$. No fungicides were added to the control flask (0.0). The experiments were carried out in 2-3-fold repetition. We used deliberately low concentrations of a.s. to get the growth of the fungus even at the most effective a.s. ratios.

Mycelium was grown for 14 days. Then the culture fluid and mycelium were filtered through pre-dried at a temperature of $55^{\circ} \mathrm{C}$ and weighted paper filters (white or red tape). The resulting filters with mycelium were dried at a temperature of $55^{\circ} \mathrm{C}$ to constant weight, reweighed, and the mass of mycelium was calculated. The data are shown in Figs. 1 and 2.

The toxigenic effect of carbendazim, azoxystrobin and pyraclostrobin was assessed by biotest method. The culture fluid passing through the paper filter was filtered again through a Whatman ${ }^{\mathrm{TM}}$ syringe filter with a pore size of $1.6 \mu \mathrm{m}$ to exclude infection.

The surface of grains of wheat of Avesta cultivar were sterilized with ethanol in accordance to Interstate standard 12044-93 [10]. Then 15-25 sterile grains were placed in sterile Petri dishes with $10 \mathrm{ml}$ of disinfected culture fluid.

The germination power was assessed on the 4th day of germination in accordance to Interstate standard 12038-84 [11]. The amounts of normal, abnormal and dead grains were calculated. We selected grains with deliberately low seeding conditions to demonstrate the effect of fungicides and the culture medium more convincingly. The experiments were carried out in 2-3-fold repetition. The data are shown in Fig. 3.

\section{Results and discussion}

Carbendazim (methyl $\mathrm{N}-(1 \mathrm{H}-$-benzimidazol-2-yl)carbamate) is a broad-spectrum benzimidazole antifungal with potential antimitotic and antineoplastic activities. Although the exact mechanism of action is unclear, carbendazim appears to binds to an unspecified site on tubulin and suppresses microtubule assembly dynamic. This results in cell cycle arrest at the G2/M phase and an induction of apoptosis. Carbendazim is a member of the class of benzimidazoles that is 2-aminobenzimidazole in which the primary amino group is substituted by a methoxycarbonyl group. A fungicide, carbendazim controls Ascomycetes, Fungi Imperfecti, and Basidiomycetes on a wide variety of crops, including bananas, cereals, cotton, fruits, grapes, mushrooms, ornamentals, peanuts, sugarbeet, soybeans, tobacco, and vegetables. It has a role as an antinematodal drug, a metabolite, a microtubule-destabilising agent and an antifungal agrochemical [12].

Azoxystrobin (methyl (E)-2-[2-[6-(2-cyanophenoxy)pyrimidin-4-yl]oxyphenyl]-3methoxyprop-2-enoate) is an aryloxypyrimidine having a 4,6-diphenoxypyrimidine skeleton in which one of the phenyl rings is cyano-substituted at C-2 and the other carries a 2methoxy-1-(methoxycarbonyl)vinyl substituent, also at C-2. An inhibitor of mitochondrial respiration by blocking electron transfer between cytochromes $\mathrm{b}$ and $\mathrm{c} 1$, it is used widely as a fungicide in agriculture. It has a role as a mitochondrial cytochrome-bc1 complex inhibitor, a xenobiotic, an environmental contaminant, an antifungal agrochemical and a quinone outside inhibitor. It is a nitrile, an aryloxypyrimidine, an enoate ester, an enol ether, a methyl ester and a methoxyacrylate strobilurin antifungal agent. Azoxystrobin is a methoxyacrylate analog and a strobilurin fungicide [13].

Pyraclostrobin (methyl N-[2-[[1-(4-chlorophenyl)pyrazol-3-yl]oxymethyl]phenyl]-Nmethoxycarbamate) is a carbamate ester. A fungicide used to control major plant pathogens including Septoria tritici, Puccinia spp. and Pyrenophora teres. It has a role as a mitochondrial cytochrome-bc1 complex inhibitor, a xenobiotic, an environmental contaminant and an antifungal agrochemical. It is a member of pyrazoles, a carbamate ester, an aromatic ether, a member of monochlorobenzenes, a methoxycarbanilate strobilurin antifungal agent and a carbanilate fungicide [14]. 
Many fungicides are known to have a toxic effect both independently $[15,16]$ and indirectly, through their influence on the production of mycotoxins in addition to their protective effect. The choice of a.s. in our experiments is based on the fact that triazoles inhibit the production of mycotoxins [7], and strobilurins increase it [9]. Carbendazim belongs to the class of benzimidazoles, so it may also have some characteristics of triazoles.

Fig. 1 and fig. 2 demonstrates that the a.s. in different ratios had a fungistatic effect, but pyraclostrobin was more effective as compared to azoxystrobin in common. For example, the mass of the formed mycelium in medium with carbendazim at a concentration of $3 \mathrm{ppm}$ and azoxystrobin at a concentration of $1 \mathrm{ppm}$ was $32.6 \mathrm{mg}$, and the mass of mycelium in medium with carbendazim at a concentration of $3 \mathrm{ppm}$ and pyraclostrobin at a concentration of $1 \mathrm{ppm}$ was $30.7 \mathrm{mg}$ (in the control without fungicides the mass of mycelium was $43.3 \mathrm{mg}$ ).

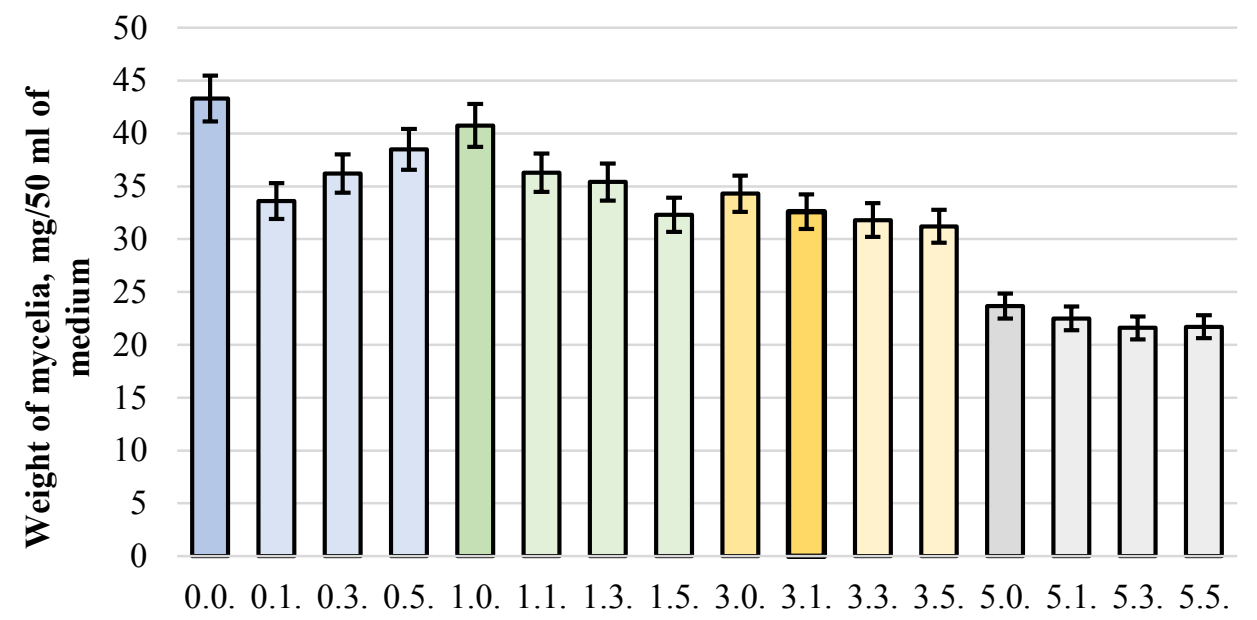

\section{Carbendazim : Azoxystrobin Ratio}

Fig. 1. The influence of carbendazim and azoxystrobin on growth of MFG58518 F. oxysporum in static culture.

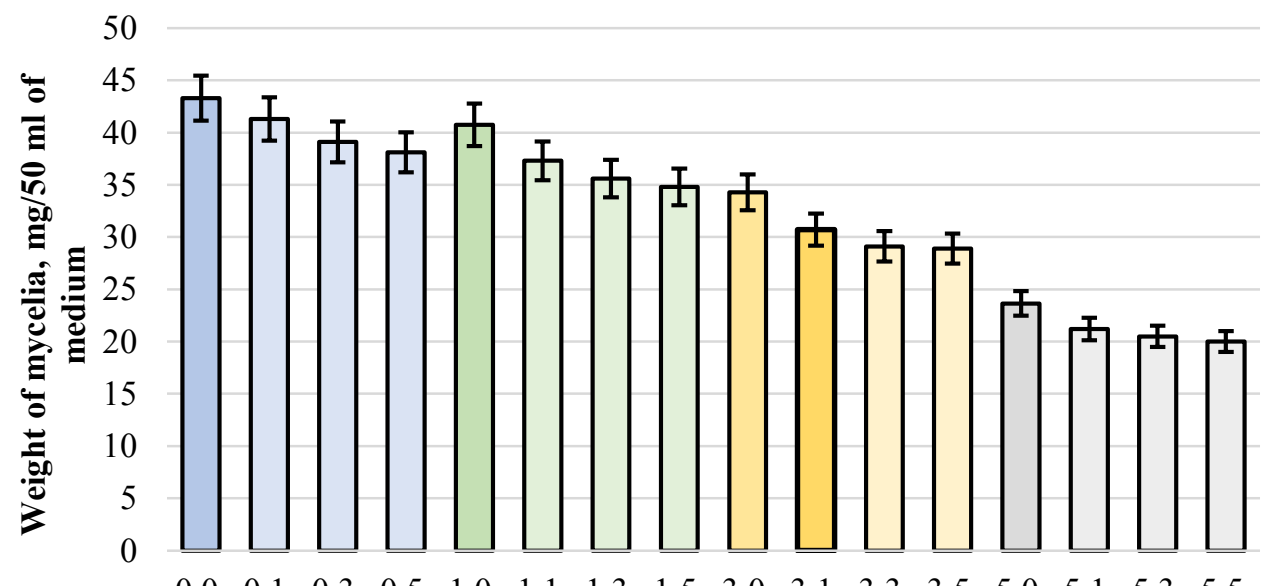

Carbendazim : Pyraclostrobin Ratio

Fig. 2. The influence of carbendazim and pyraclostrobin on growth of MFG58518 F. oxysporum in static culture. 


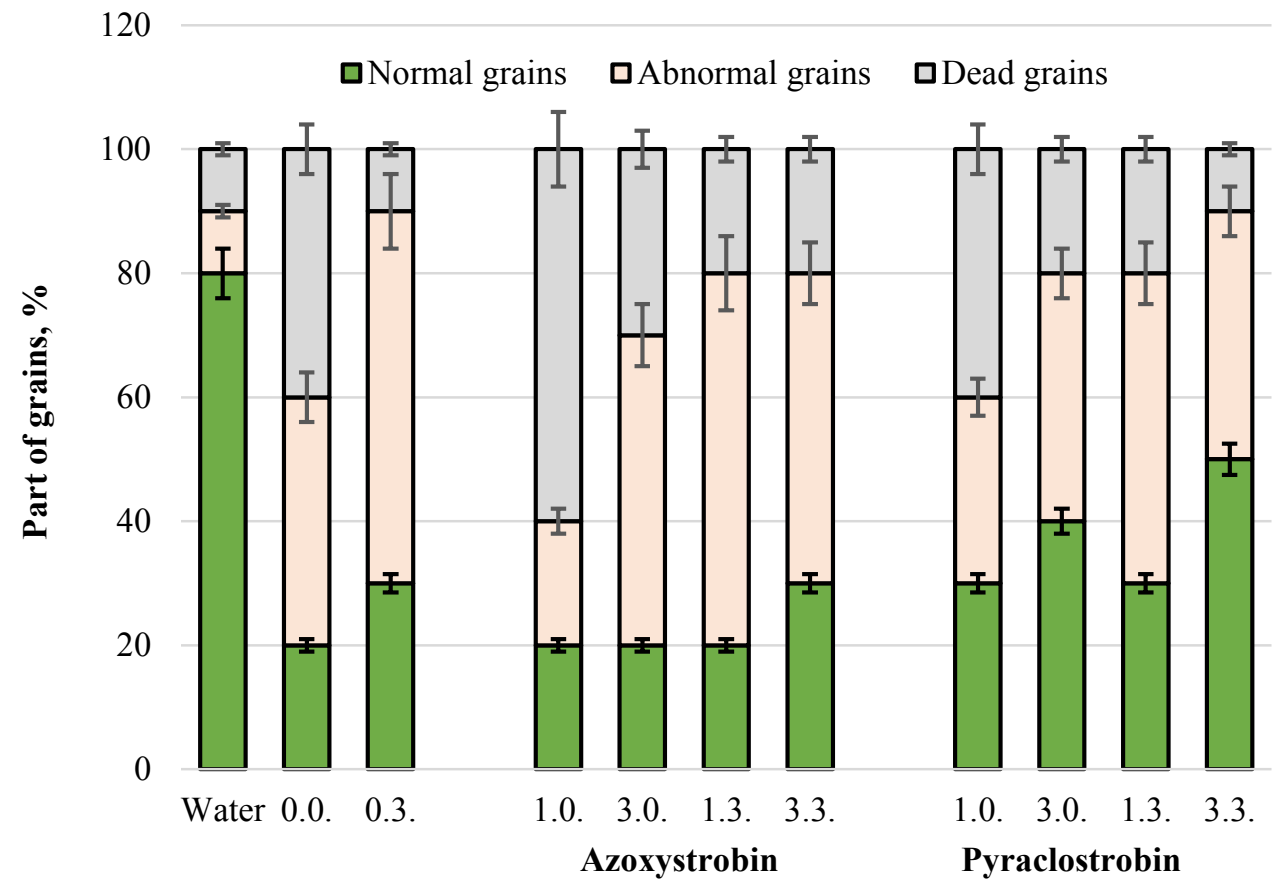

Fig. 3. The influence of culture fluid of MFG58518 F. oxysporum on germination power of wheat.

Toxigenic properties of named strain are shown in Fig. 3. Even without the use of fungicides (0.0), the culture fluid decreased the germination power of grains. Carbendazim weakened the toxigenic properties of the strain. Azoxystrobin at doses of $1 \mathrm{ppm}$ and $3 \mathrm{ppm}$ did not decrease the toxigenic properties of the strain, and pyraclostrobin at doses of 1 and 3 ppm significantly reduced the toxigenic properties of the strain. Mixtures of carbendazim and azoxystrobin (1:3 and 3:3) reduced the toxigenic properties of the strain, but their effect was lesser than the effect of mixtures of carbendazim and pyraclostrobin (1:3 and 3:3).

The culture fluid after the cultivation of $F$. oxysporum with addition of azoxystrobin and pyraclostrobin in small doses (1:0 ratio) was toxic for grains. It promoted the acute mortality. the death of a significant part of the grains (Fig. 3) was noted in it. The fungistatic effect of those doses was not expressed (Fig. 1,2). Therefore, the adaptation of the fungus was realized as a change in its metabolism.

An increase in the dose of azoxystrobin decreased the mortality of seeds, and an increase in the dose of pyraclostrobin increased the germination power (3:0, Fig. 3). These doses of fungicides had a fungistatic effect (Fig. 1, 2). The addition of carbendazim increased the fungistatic effect and decreased the toxicity of the strain.

\section{Conclusion}

The presence of mycotoxins in the culture medium of $F$. oxysporum was the reason of the inhibition of germination power of seeds. The choice of a.s. and the development of combined fungicides requires to consider a possible phytotoxic effect. That effect depends on the properties and dose of the a.s. on the one hand, and on the properties of phytopathogenic fungi on the other hand. Fungicides are effective against plant diseases. Azoxystrobin, pyraclostrobin and carbendazim are well known and widely used plant protection products. They demonstrated effectiveness against the studied strain of $F$. oxysporum. However, small doses of a.s. promoted the accumulation of substances in the 
culture fluid, which leaded to the death of part of the grains and inhibition of the germination process. Development of combined fungicides with sufficient doses of active substances reduces the risk of inhibition of the growth of the protected crops.

\section{References}

1. T. Yu. Gagkaeva, O. P. Gavrilova, I. V. Shamshev, O. G. Selitskaya, E. I. Savelieva, Fusarium - mycotoxins, taxonomy, genomics, biosynthesis, pathogenicity, resistance, disease control, 109 (INRA Research Unit MycSA, France, 2013)

2. N. Venkatesh, N. P. Keller, Front. in Microbiol. 10, 403 (2019)

3. I. N. Voronchihina, V. S. Rubec, Izvestiya of Timiryazev Agricultural Academy 3, 17 (2018). DOI: 10.26897/0021-342X-2018-3-17-32

4. G. P. Kononenko, A. A. Burkin, O. P. Gavrilova, T. Y. Gagkaeva, Agricult. and Food Sci. 24, 323 (2015)

5. F. Cheli, L. Pinoti, A. Campagnoli, E. Fusi, R. Rebuci, A. Baldi, Italian J of Food Sci. 20, 447 (2008)

6. Sanitary Rules and Regulations 2.3.2.1078-01 Hygienic requirements for the safety and nutritional value of food products (as amended on July 6, 2011) (Moscow, 2011)

7. M. Beyer, M. B. Klix, H. Klink, J.-A. Verreet, J. of Plant Dis. and Prot. 113, 241 (2006)

8. D. S. Syrova, A. I. Shaposhnikov, N. M. Makarova, T. Yu. Gagkaeva, I. A. Khrapalova, V. V. Emelyanov, Yu. V. Gogolev, Ph. B. Gannibal, A. A. Belimov, Mycol. and Phytopath. 53, 301 (2019)

9. F. M. Ellner, Mycotoxin Res. 21, 112 (2005). DOI: 10.1007/BF02954432

10. GOST 12044-93 Mezhgosudarstvennyj standart. Semena sel'skohozyajstvennyh kul'tur. Metody opredeleniya zarazhennosti boleznyami [Interstate standard 12044-93 Seeds of agricultural crops. Methods for determination of diesease infestation] (Standardinform, Moscow, 2011)

11. GOST 12038-84 Mezhgosudarstvennyj standart. Semena sel'skohozyajstvennyh kul'tur. Metody opredeleniya vskhozhesti [Interstate standard 12038-84 Seeds of agricultural crops. Methods for determination of germination (Standardinform, Moscow, 2011)

12. National Center for Biotechnology Information. PubChem Database. Carbendazim, $C I D=25429$ (https://pubchem.ncbi.nlm.nih.gov/compound/Carbendazim)

13. National Center for Biotechnology Information. PubChem Database. Azoxystrobin, $C I D=3034285$ (https://pubchem.ncbi.nlm.nih.gov/compound/3034285)

14. National Center for Biotechnology Information. PubChem Database. Pyraclostrobin, $C I D=6422843$ (https://pubchem.ncbi.nlm.nih.gov/compound/6422843)

15. E. V. Baibakova, E. E. Nefedjeva, M. Suska-Malawska, M. Wilk, G. A. Sevriukova, V. F. Zheltobriukhov, Annual Research \& Review in Biology 32, 1 (2019). DOI: 10.9734/arrb/2019/v32i330083

16. E. V. Baybakova, E. E. Nefed'eva, S. L. Belopukhov, Proceedings of Universities. Applied Chemistry and Biotechnology 6, 57 (2016). DOI: 10.21285/2227-2925-2016-63-57-64 